\title{
De las facilidades para el pago de las deudas tributarias en tiempos de covid-19 en Perú y Colombia
}

\author{
Of the facilities for the payment of tax \\ debts in Covid-19 Time in Peru and \\ Colombia
}

Das instalações para o pagamento de dívidas fiscais no tempo Covid-19 no Peru y Colombia

MERY LÓPEZ NIEVES*

* Contadora Pública; especialista en Revisoría Fiscal y Auditoría Externa; magíster en Tributación Internacional, Comercio Exterior y Aduanas de la Universidad Externado de Colombia. Actualmente se desempeña como funcionaria pública de la Dirección de Impuestos y Aduanas Nacionales. meloni424@hotmail.com / https://orcid.org/0000-0002-0605-8989 DoI: https://doi.org/10.18601/16926722.n18.10 


\title{
Resumen
}

La pandemia del covid-19 ha afectado de manera negativa la economía a nivel global, lo que ha llevado a que los contribuyentes no puedan asumir el pago de sus obligaciones tributarias como lo harían en condiciones normales; en este contexto, el artículo analiza los cambios normativos que Perú ha realizado en su modelo de facilitación del pago de los impuestos, a partir de las nuevas expectativas de recaudo tributario, en el marco de los efectos del covid-19, de modo comparativo con Colombia.

Palabras clave: facilidades de pago; Colombia; Perú; impuestos; covid-19.

\begin{abstract}
The CoviD-19 pandemic has negatively affected the economy at a global level, which has led to taxpayers not being able to assume the payment of their tax obligations as they would under normal conditions. In this context, this article analyzes the changes regulations that Peru has carried out in its tax payment facilitation model, based on the new expectations of tax collection in the framework of the effects of Covid-19, in a comparative way with Colombia.
\end{abstract}

Key words: Payment facilities; Colombia; Peru; taxes; Covid-19.

\section{Resumo}

A pandemia do CoviD-19 afetou negativamente a economia em nível global, o que levou os contribuintes a não poderem assumir o pagamento de suas obrigações fiscais como fariam em condições normais.Neste contexto, este artigo analisa as mudanças regulamentos que o Peru adotou em seu modelo de facilitação de pagamento de impostos, com base nas novas expectativas de arrecadação de impostos no âmbito dos efeitos do Covid-19, de forma comparativa com a Colômbia.

Palavras-chave: Meios de pagamento; Colômbia; Peru; impostos; Covid-19. 


\section{Introducción}

El 2020 pasará a la historia como el año en que el mundo, como en ningún otro tiempo, fue protagonista y testigo en primera fila -y de manera simultánea gracias a las tecnologías de información y la comunicación (TIC) - de las grandes afectaciones que una pandemia bautizada covid-19 tuvo en la humanidad.

El coronavirus trascendió el ámbito de la salud, impactó violentamente lo social y lo económico, lo que de manera inevitable obligó a los gobiernos a tomar posición para afrontar la crisis y los efectos esperados.

De esta manera, podemos distinguir dos líneas en la toma de decisiones de los Estados en latinoamerica. Una, la de aquellos países en donde se privilegió la importancia de la economía y la necesidad de mantener el aparato productivo en marcha, no obstante las previsibles pérdidas humanas. La otra, la de quienes le apostaron a salvaguardar el capital humano, decretando un aislamiento que permitiera preparar las clínicas y los hospitales de nuestros deprimidos sistemas de salud, para tener una mayor probabilidad de afrontar con dignidad el pico de la pandemia.

De todas formas, los países se obligaron a revisar sus políticas económicas y fiscales aplicables en el corto y mediano plazo, con la intención de implementar estrategias que viabilicen el futuro socioeconómico, para lo cual expidieron normas tributarias enmarcadas en la emergencia sanitaria y económica.

En Latinoamérica, Perú y Colombia no han escapado a esta situación y de manera coherente con el momento que estamos viviendo, sus administraciones tributarias se han dado a la tarea de facilitar y viabilizar el pago de los impuestos de los contribuyentes teniendo en cuenta la prioridad que establece el ser humano en la satisfacción de sus necesidades básicas, pero atendiendo también al hecho de que los Estados no pueden cumplir su función social, garantizar y satisfacer las necesidades básicas en salud, educación e infraestructura de sus pueblos si no se hace el necesario recaudo de los tributos.

En este contexto, analizaremos los cambios normativos que Perú ha realizado en su modelo de facilitación del pago de los impuestos, a partir de las nuevas expectativas de recaudo tributario en el marco de los efectos del Covid-19, en modo comparativo con Colombia.

\section{Facilidades de pago en Perú}

Antes de la expedición del Decreto Supremo 044-2020-PCM mediante el cual fue declarado en Perú el Estado de Emergencia Nacional (en principio por un término de quince días calendario), los contribuyente podían acogerse a un aplazamiento o fraccionamiento (facilidad de pago) de sus deudas tributarias al tenor de lo dispuesto en la Resolución 161-2015 "Reglamento de aplazamiento y/o fraccionamiento de la deuda tributaria por

Reglamentario del artículo $36^{\circ}$ del Código Tributario Peruano. 
tributos internos", expedida por la Superintendencia Nacional de Aduanas y de Administración Tributaria (SUNAT) y vigente desde el 15 de julio de 2015.

La norma en comento prevé el procedimiento para acogerse al aplazamiento o fraccionamiento de las deudas tributarias originadas en los tributos administrados por la SUNAT, incluyendo las deudas por regalía minera y el gravamen especial a la minería, con excepción de los impuestos retenidos o percibidos.

Por norma general, el procedimiento se inicia (al igual que en Colombia) con una solicitud que se realiza con el diligenciamiento del formulario virtual 687 a través del sistema informático de la SUNAT. El contribuyente, previamente y de manera opcional, puede gestionar el reporte de precalificación, el cual le permite evaluar si cumple los requisitos exigidos para acogerse al aplazamiento o fraccionamiento de la deuda tributaria.

Ahora bien, las deudas que en Perú pueden ser objeto del aplazamiento o fraccionamiento son las que se originen en los tributos administrados por la SUNAT, así como las administradas en la contribución al Fondo Nacional de Vivienda (FONAVI).

Para el acogimiento deberán cumplirse los siguientes presupuestos:

i) En el caso de personas naturales obligadas a presentar la declaración jurada anual del impuesto a la renta, la solicitud de aplazamiento o fraccionamiento de la regularización de dicho tributo por rentas de capital o trabajo puede efectuarse inmediatamente después de la presentación de la declaración en comento, a través de un enlace que le genera el sistema al contribuyente, para realizar la solicitud.

Si el contribuyente no ejerce la opción antes descrita, puede realizar la solicitud de manera ordinaria trascurridos cinco días hábiles desde la fecha de presentación de la declaración jurada anual del impuesto a la renta.

ii) En el caso de personas naturales no obligadas a presentar declaración jurada anual del impuesto a la renta por rentas del trabajo (contribuyentes pertenecientes al Nuevo régimen único simplificado [NRUS] ${ }^{2}$ y contribuyentes del Régimen especial de Impuesto a la Renta $[\mathrm{RER}]^{3}$ ), la solicitud de aplazamiento o fraccionamiento de la regularización de dicho tributo puede efectuarse a partir del día siguiente al vencimiento del plazo para el pago del referido impuesto.

iii) Para los contribuyentes cuyos ingresos se originen en los caracterizados en la tercera categoría ${ }^{4}$, la solicitud de aplazamiento o fraccionamiento del impuesto a la renta puede presentarse a partir del primer día hábil del mes de mayo del ejercicio en el cual se

2 Se distinguen dos categorías: Categoría 1. Topes de ingresos y compras hasta 5.000 soles; Categoría 2. Topes de ingresos y compras que no superen los $\mathrm{S} / 8.000$.

3 Contribuyentes personas naturales y jurídicas domiciliadas en Perú, cuyos ingresos y compras no superen los S/525.000 y activos no superiores a S/126.000 (sin incluir predios y vehículos).

4 La tercera categoría de impuesto a la renta grava ingresos que provengan de las personas naturales con negocio, las rentas que obtengan las personas jurídicas, y las rentas que expresamente la Ley del Impuesto a la Renta ha clasificado en esta Categoría 3a. 
produce su vencimiento, siempre que hayan transcurrido cinco (5) días hábiles desde la presentación de la referida declaración.

Adicionalmente, podrán ser objeto de fraccionamiento los intereses generados una vez vencido el plazo para la regularización de la declaración o pago del impuesto sobre la renta, las deudas generadas en tributos derogados, y las deudas tributarias originadas en el incumplimiento de las cuotas de deudas acogidas a los beneficios de la Ley 27344 - Régimen Especial de Fraccionamiento Tributario (REFT) ${ }^{5}$, los saldos pendientes de deudas acogidas al Decreto Legislativo 914 - Sistema Especial de Actualización y Pago de Deudas Tributarias (SEAP), o las cuotas incumplidas en deudas acogidas al beneficio de la Ley 27681- Ley de reactivación a través del sinceramiento de las deudas tributarias (RESIT); en estos casos, el acogimiento al aplazamiento o fraccionamiento debe realizarse por la totalidad de las órdenes de pago que contengan las cuotas pendientes de pago.

No obstante lo anterior, en Perú existen deudas tributarias que no pueden ser objeto de aplazamiento o fraccionamiento, entre otras: las correspondientes al último periodo tributario vencido a la fecha de presentación de la solicitud; aquellas cuyo vencimiento se produzca en el mes de presentación de la solicitud (salvo algunas excepciones) ; el impuesto temporal a los activos netos (ITAN); las que hubieran sido materia de aplazamiento o fraccionamiento anterior (con algunas excepciones); las que se encuentran en procesos de reestructuración patrimonial al amparo de la Ley 27809 - Ley General del Sistema Concursal; los tributos retenidos o percibidos; las que se encuentran en trámite de reclamación, apelación, demanda contenciosa administrativa o estén comprendidas en acciones de amparo, salvo que a la fecha de presentación de la solicitud la SUNAT hubiera aceptado el desistimiento de la pretensión y este conste en resolución en firme.

En el marco de posibilidades antes examinado, los contribuyentes en Perú pueden acogerse al aplazamiento o fraccionamiento de sus deudas tributarias atendiendo los plazos establecidos, así: i) para el caso de los aplazamientos el plazo mínimo es un mes y el máximo es 6 meses, ii) para el caso del fraccionamiento el plazo mínimo es de 2 meses y el plazo máximo es de 72 meses, y iii) para los casos de aplazamiento y fraccionamiento (cuando se otorgan de manera conjunta), el mínimo del aplazamiento es de un mes, el mínimo de fraccionamiento es 2 meses y el plazo máximo es de 72 meses.

La cuota de acogimiento se determina de acuerdo con el monto y plazo de la deuda tributaria que se solicita fraccionar o aplazar y fraccionar, no puede ser menor al $5 \%$ de la unidad impositiva tributaria (UIT), y debe cancelarse a la fecha de presentación de la

5 Ley 27344. Régimen Especial de Fraccionamiento Tributario-Reft. Aplicable a Deudores tributarios que hubieran acumulado dos o más cuotas vencidas o pendientes de pago a 30 de agosto de 2000 , y la deuda que no se encuentre acogida al beneficio mencionado (fraccionamiento), de los tributos recaudados o administrados por la Superintendencia Nacional de Administración Tributaria (SUNAT), incluidas las que vienen del ex-Fondo Nacional de Vivienda (FONAVI), el Servicio Nacional de Capacitación para la Industria de la Construcción (SENCICO), el Servicio Nacional de Adiestramiento en Trabajo Industrial (SENATI), la Superintendencia Nacional de Aduanas (aduanas), la Oficina de Normalización Previsional (ONP) y el Seguro Social de Salud (ESSALUD). 
solicitud de fraccionamiento, o hasta la fecha de vencimiento de pago de los intereses del aplazamiento tratándose de solicitudes de aplazamiento y fraccionamiento (tabla 1).

Tabla 1.

\begin{tabular}{|c|c|c|}
\hline Monto de la deuda & Plazo (meses) & Cuota de acogimiento (\%) \\
\hline \multirow{4}{*}{ Hasta 3 uIT* } & Hasta 12 & 0 \\
\cline { 2 - 3 } & De 13 a 24 & 6 \\
\cline { 2 - 3 } & De 25 a 36 & 8 \\
\cline { 2 - 3 } & De 37 a 48 & 10 \\
\cline { 2 - 3 } & De 49 a 60 & 12 \\
\hline \multirow{5}{*}{ Mayor a 3 uIT } & De 61 a 72 & 6 \\
\cline { 2 - 3 } & Hasta 24 & 8 \\
\cline { 2 - 3 } & De 25 a 36 & 10 \\
\cline { 2 - 3 } & De 37 a 48 & 12 \\
\cline { 2 - 3 } & De 49 a 60 & 14 \\
\hline
\end{tabular}

* UIT = Unidad Impositiva Tributaria.

Fuente: elaboración propia.

No obstante lo anterior, la norma prevé casos en los cuales no es exigible la cuota de acogimiento, así: 1) para la presentación de las solicitudes de aplazamiento, 2) cuando el deudor tributario tenga la calidad de buen contribuyente a la fecha de la solicitud y 3) cuando el plazo y el monto de la deuda que se solicita fraccionar o aplazar y fraccionar es menor o igual a 12 meses y menor o igual a 3 UIT, respectivamente.

El deudor tributario en Perú deberá ofrecer garantías para acogerse al fraccionamiento de la obligación en los siguientes casos:

- Cuando sea una persona natural que con antelación a la fecha de la presentación de la solicitud tenga proceso penal en trámite por delito tributario o sentencia condenatoria consentida o ejecutoriada vigente por dicho delito.

- Cuando se trate de una persona jurídica cuyo representante legal tiene proceso penal en trámite por delito tributario o sentencia condenatoria ejecutoriada vigente por dicho delito, con anterioridad a la fecha de presentación de la solicitud.

- Cuando la solicitud de fraccionamiento sea para un contrato de colaboración empresarial que lleva contabilidad independiente, inscrito en el RUC con tal condición.

- Cuando el total del monto por fraccionar (incluidas las deudas tributarias, los refinanciamientos, los intereses y demás) menos la cuota de acogimiento supere las 100 UIT. En este caso el monto por garantizar es la parte que excede las 100 UIT. 
El deudor tributario puede otorgar dos clases de garantías: 1) la Carta fianza, que es un contrato o póliza de garantía de cumplimiento de pago de la deuda (emitida por las empresas del sistema financiero y empresas del sistema de seguros autorizadas por la Superintendencia de Banca), cuyo respaldo debe superar en un $5 \%$ el valor total de las obligaciones objeto del fraccionamiento, y 2) hipoteca de primer rango, que debe superar en un $40 \%$ el monto total de la obligación por fraccionar.

En virtud de lo anterior, se pueden ofrecer u otorgar tantas garantías como sean necesarias para cubrir la deuda que se va a garantizar, contenida en la solicitud hasta su cancelación, aun cuando concurran garantías de distinta clase.

Una vez aprobada la solicitud por parte de la SUNAT, los pagos de las cuotas mensuales que se efectúen en cumplimiento del fraccionamiento se imputarán en primera instancia a los intereses moratorios y después a la amortización de la deuda tributaria. Los pagos de las cuotas se realizarán a través del sistema pago fácil, mediante sUNAT Virtual o en los bancos habilitados utilizando el NPS, los cuales generan el formulario 1662.

La norma también ha previsto, en su artículo 21 , las situaciones en las cuales se pierde el beneficio del otorgamiento del aplazamiento o fraccionamiento, así:

- En caso de fraccionamiento cuando acumule dos cuotas consecutivas sin pago.

- Cuando no se cumpla con el pago de la última cuota dentro del plazo establecido para su vencimiento.

- En caso de solo aplazamiento, cuando no cumpla con pagar la totalidad de la deuda tributaria aplazada, más el interés correspondiente al vencimiento del plazo concedido.

- En caso de aplazamiento y fraccionamiento se pierde: i) ambos cuando no se paguen los intereses del aplazamiento hasta la fecha de su vencimiento; ii) el fraccionamiento, cuando no cancele la cuota de acogimiento de acuerdo con lo señalado en el literal b) del artículo $9^{\circ}$ del reglamento (RS 161-2015) o si habiendo cumplido con pagar la cuota de acogimiento y la totalidad del interés del aplazamiento, se dejaren de pagar dos cuotas consecutivas, o se incumpla con el pago de la totalidad de la última cuota dentro del término de su vencimiento.

- Cuando el deudor tributario no cumpla con mantener las garantías otorgadas a favor de la SUNAT o no las renueve (en los casos establecidos en el reglamento).

Es de destacar, que la pérdida del aplazamiento o fraccionamiento se determina con base en las causales previstas en el reglamento vigente al momento de la emisión de la resolución que declare la misma.

Teniendo en cuenta el impacto que el Estado de Emergencia Nacional podía ocasionar en los sectores económicos a los que se les aplica el Sistema de Pago de Obligaciones Tributarias, la SUNAT expidió el 18 de marzo de 2020, la RS 058-2020 cuyo artículo 2 reemplazó algunas de las causales de pérdida de aplazamiento o fraccionamiento y del refinanciamiento contenidas en los literales a), b) y c) del artículo 21 de la Resolución 
de Superintendencia 161-2015 y en los literales a),b) y c) del artículo 21 de la Resolución de Superintendencia 190-2015 de la SUNAT.

El citado artículo $2^{\circ}$, en términos generales, dispone que a los deudores tributarios que a corte 15 de marzo de 2020 la SUNAT no les hubiere notificado resolución declaratoria de pérdida del aplazamiento o fraccionamiento o refinanciamiento, se les extienden los plazos cuyo vencimiento era el 31 de marzo de 2020, hasta el 30 de abril de 2020 para la pérdida de los beneficios contemplados en los literales a), b) y c) de los artículos 21 de las resoluciones de Superintendencia 161-2015 y 190-2015 de la SUNAT.

En Perú, mediante Decreto Supremo 051-2020-PCM, se prorrogó el estado de emergencia nacional, la administración tributaria igualmente amplió las medidas para facilitar el cumplimiento de las obligaciones tributarias mediante la RS 065-2020/SUNAT, cuyo artículo 3 modificó el artículo 2 de la RS 0058-2020/SUNAT, quedando el artículo 21 de las mencionadas resoluciones con las disposiciones para la pérdida de los beneficios, tal como se indica a continuación:

1. El fraccionamiento se pierde:

- Cuando se adeude el íntegro de dos cuotas, sin computar para estos efectos las cuotas que vencían el 31 de marzo y 30 de abril de 2020, siempre que estas se hayan pagado con sus respectivos intereses moratorios hasta el 29 de mayo de 2020.

- Cuando no se pague el total de la última cuota dentro del plazo del vencimiento. Si la fecha del vencimiento de la última cuota era el 31 de marzo o 30 de abril de 2020, no se pierde el beneficio si el pago se efectúa hasta el 29 de mayo de 2020 incluidos los intereses moratorios correspondientes.

2. En caso de solo aplazamiento, este cesa en el evento del no pago de la deuda tributaria aplazada con sus respectivos intereses al vencimiento del plazo concedido. Si el plazo otorgado vencía el 31 de marzo o el 30 de abril de 2020, este término se extiende hasta el 29 de mayo de 2020.

3. Para los casos de aplazamiento con fraccionamiento, la nueva norma contempló que: i) se pierden ambos, cuando el deudor tributario no pague la totalidad del interés del aplazamiento hasta la fecha de su vencimiento. Si la fecha de vencimiento es el 31 de marzo o el 30 de abril de 2020, se pierden ambos cuando no se cumpla con pagar el referido interés hasta el 29 de mayo de 2020; ii) se pierde el fraccionamiento cuando no se cancele la cuota de acogimiento en las fechas previstas para ello; no obstante, si dicha cuota debía pagarse el 31 de marzo o el 30 de abril de 2020, este plazo (igual que en los casos anteriores) se extiende hasta el 29 de mayo de 2020.

El beneficio del fraccionamiento también se desconoce en los casos en que, habiendo pagado la cuota de acogimiento y el interés del aplazamiento, se acumule deuda de dos 
cuotas consecutivas del fraccionamiento. En estos casos, la cuota que vencía el 31 de marzo o el 30 de abril de 2020 no se computa para efecto de lo dispuesto en la norma siempre que dicha cuota y los intereses moratorios que correspondan, se paguen hasta el 29 de mayo de 2020 .

Asimismo, la regulación contempla que el fraccionamiento cesa si no se paga la última cuota dentro del plazo del vencimiento; si la última cuota se vencía el 31 de marzo o el 30 de abril de 2020, el beneficio no se pierde siempre y cuando el deudor tributario pague la totalidad de la cuota hasta el 29 de mayo de 2020.

Vale anotar, que las modificaciones efectuadas por la RS 0065-2020/SUNAT no tienen aplicabilidad para los aplazamientos o fraccionamientos o refinanciamientos a los que se les aplican las causales de pérdida previstas en el artículo 13 de la RS 051-2019\%/SUNAT y en el artículo 11 de la RS 100-2017/SUNAT.

\section{Facilidades de pago en Colombia}

En Colombia, el otorgamiento de facilidades de pago de los tributos está contenido en el artículo 814 del Estatuto Tributario (ET), y en él se prevé que, mediante resolución, los administradores de Impuestos Nacionales pueden conceder facilidades de pago al deudor o a un tercero a su nombre por un plazo máximo de cinco años para el pago de todos los impuestos administrados por la Dirección General de Impuestos Nacionales (DIAN), así como los intereses y las sanciones que de ellos se originen.

Para tales efectos, los deudores (o un tercero a su nombre) pueden garantizar el pago de las deudas tributarias con varias modalidades, a saber: fideicomiso de garantía, bienes para su embargo y secuestro, garantías personales (en deudas que no superen las 3.000 UVT), garantías reales, bancarias o de compañías de seguros, o cualquiera otra garantía que a juicio de la Administración respalde suficientemente la deuda.

Las facilidades de pago concedidas a un contribuyenye antes de entrar en vigencia la Ley 2010 de 2019 debían contener la totalidad de las obligaciones tributarias que tuviere el contribuyente pendientes de pago a la fecha de suscribir dicha facilidad de pago.

Con ocasión de la Ley 2010 de 2019, que entró en vigencia para el año gravable 2020, se realizaron algunas modificaciones al procedimiento y los requisitos establecidos para el otorgamiento de las facilidades de pago para el caso de las obligaciones respecto de las cuales el contribuyente solicitó la aplicación del principio de favorabilidad en la etapa de cobro (art. 640, pará. $5^{\circ}, \mathrm{ET}$ ).

Para acogerse a este beneficio, el deudor tributario deberá suscribir la facilidad hasta el 30 de junio de 2020 (ampliado hasta el 30 de noviembre, art. $3^{\circ}$ Decr. 688 de 2020) y el

6 Resolución de la Superintendencia que aprueba facilidades para deudores tributarios de zonas declaradas en estado de emergencia por desastres naturales.

7 Resolución de la Superintendencia que aprueba facilidades para deudores tributarios de zonas declaradas en estado de emergencia por desastres naturales ocurridos desde enero de 2017. 
plazo máximo para el pago de las obligaciones cobijadas es de doce meses a partir de la suscripción del acuerdo, en virtud de lo cual el deudor decide cuáles obligaciones desea incluir en la facilidad, además de aquella a la que se le aplicó el principio de favorabilidad.

Para obtener esta facilidad, el deudor no requiere aportar los estados financieros, ni el flujo de caja del periodo de vigencia del acuerdo de pago, como tampoco tendrá que entregar certificados de ingresos firmados por contadores públicos o revisores fiscales.

La tasa de interés aplicable a estas facilidades es el interés bancario corriente más dos puntos porcentuales y, en cuanto a las garantías, basta relacionarlas y aportar el correspondiente avalúo.

Ahora bien, en el marco de la declaratoria de emergencia económica, social y ecológica declarada en Colombia, por el término de 30 días, mediante el Decreto 637 del 6 de mayo de 2020, y con el fin de estimular la presentación y el pago de las declaraciones tributarias, aún en medio de los consecuentes efectos económicos originados en las medidas de aislamiento tomadas en desarrollo de la pandemia por el covid-19, el Gobierno nacional expidió el Decreto 688 del 22 de mayo de 2020, en cuyo artículo 2 se introdujo la modalidad de "Facilidades de pago abreviadas", aplicable a los contribuyentes que presenten sus declaraciones tributarias por los impuestos administrados por la DIAN, entre el $1^{\circ}$ de abril y el $1^{\circ}$ de julio de 2020 y que estén en mora en el pago, con fecha límite de solicitud hasta el 6 de agosto de 2020.

Una vez efectuada la solicitud por parte del contribuyente, la DIAN deberá pronunciarse en un término no superior a 15 días, y la facilidad o acuerdo de pago será otorgada por un plazo máximo de 12 meses, no requiere garantía real y el interés del plazo será el interés bancario corriente.

Para la obtención de la aprobación del acuerdo de pago, el representante legal de la sociedad deberá aportar, con la solicitud, una certificación-declaración juramentada en la que se demuestre la necesidad de caja de la empresa y las proyecciones financieras que viabilizarán el pago de la obligación dentro del plazo de 12 meses.

Finalmente, el incumplimiento de la facilidad dará lugar a iniciar el cobro coactivo de la obligación tributaria objeto del acuerdo, para lo cual la resolución de otorgamiento del acuerdo de pago presta mérito ejecutivo para dicho cobro. En este caso, la tasa de interés moratorio que se le aplicará a la obligación será la contemplada en el artículo 635 del ET. ${ }^{8}$

\section{Conclusiones}

Cuando comparamos las medidas adoptadas por Colombia y Perú para el manejo de la crisis económica originada en las medidas de aislamiento ocasionadas por el covid-19, dentro del contexto específico del pago de las obligaciones tributarias observamos que las medidas que toma Colombia están orientadas a las personas jurídicas, con el ánimo de 
permitirles cumplir con sus obligaciones formales de presentación de las declaraciones (incluso las de años o periodos gravables vencidos) y cobijarlas con un acuerdo de pago de trámite abreviado, con el fin de que el contribuyente no se desprenda (en tiempos de la pandemia) de recursos económicos que le permitan afrontar otras urgencias de caja.

Para estos casos, la administración ha detraído requisitos como las garantías, y solo basta la certificación firmada bajo la gravedad de juramento por parte del representante legal; ha disminuido la tasa de interés de mora aplicable, así como el tiempo de aprobación de la solicitud.

Adicionalmente, se extendió el plazo para permitir que quienes se acojan a la favorabilidad de la reducción de las sanciones disminuidas en la Ley 1819 de 2016, puedan hacerlo hasta el 30 de noviembre de 2020, lo que permitirá, entre tanto, que los contribuyentes fluyan con lo que tienen en caja.

En Perú, en lo que tiene que ver con la facilitación del pago de las deudas tributarias, el Gobierno únicamente extendió las fechas límites de pago de las cuotas correspondientes a los incumplimientos que originen la pérdida del aplazamiento o fraccionamiento o refinanciamiento, pero sin tocar la tasa de interés, los mecanismos de garantía del cumplimiento de las obligaciones de tales acuerdos, ni los tiempos de respuesta de la administración tributaria.

\section{Referencias}

Piza Rodríguez, J. R. (2015). La obligación Tributaria y sus fundamentos constitucionales. Universidad Externado de Colombia.

\section{Normatividad}

Decreto Supremo 044-2020-PCM. "Decreto Supremo que declara Estado de Emergencia Nacional por las graves circunstancias que afectan la vida de la Nación a consecuencia del brote del covid-19”, 15 de marzo de 2020. https://www.gob.pe/ institucion/pcm/normas-legales/460472-044-2020-pcm

Decreto Supremo 051-2020-PCM. "Prórroga del Estado de Emergencia Nacional declarado mediante Decreto Supremo 044-2020-PCM”, 27 de marzo de 2020. https:// www.gob.pe/institucion/pcm/normas-legales/462808-051-2020-pcm

Decreto Supremo 133-2013-EF. “Aprueba el texto único ordenado del Código Tributario", 22 de junio de 2013. http://www.sunat.gob.pe/legislacion/codigo/

http://www.sunat.gob.pe/legislacion/superin/2004/indices/indcor.htm 
Régimen Especial de Fraccionamiento Tributario - Ley 27344. www.sunat.gob.pe > legislacion > fraccion > ley27344

República de Colombia (1989). Decreto 624 de 1989. "Por el cual se expide el Estatuto Tributario de los impuestos administrados por la dirección general de impuesto nacionales". https://estatuto.co/

República de Colombia (2016). Ley 1819 de 2016. "Por medio de la cual se adopta una reforma tributaria estructural, se fortalecen los mecanismos para la lucha contra la evasión y la elusión fiscal, y se dictan otras disposiciones". 29 de diciembre de 2016. DO 50.101. http://www.secretariasenado.gov.co/senado/basedoc/ ley_1819_2016.html

República de Colombia (2019). Ley 2010 de 2019. "Por medio de la cual se adoptan normas para la promoción del crecimiento económico, el empleo, la inversión, el fortalecimiento de las finanzas públicas y la progresividad, equidad y eficiencia del sistema tributario, de acuerdo con los objetivos que sobre la materia impulsaron la Ley 1943 de 2018 y se dictan otras disposiciones". 27 de diciembre de 2019. DO 51.179.http://www.secretariasenado.gov.co/senado/basedoc/ley_1819_2016.html

República de Colombia (2020). Decreto 637 del 6 de mayo de 2020. "Por el cual se declara un Estado de Emergencia Económica, Social y Ecológica en todo el territorio nacional". https://dapre.presidencia.gov.co/normativa/normativa/DECRETO \%20 $637 \%$

República de Colombia (2020). Decreto 688 del 22 de mayo de 2020. "Por el cual se adoptan medidas tributarias transitorias en el marco del Estado de Emergencia Económica, Social y Ecológica de conformidad con el Decreto 637 de 2020”. https:// dapre.presidencia.gov.co/normativa/normativa/DECRETO \%20688\%

Resolución de Superintendencia 058-2020/SUNAT. "Disponen medidas adicionales para favorecer la disponibilidad de recursos y otorgar otras facilidades a los deudores tributarios ante la declaratoria de emergencia nacional", 18 de marzo de 2020. https://busquedas.elperuano.pe/normaslegales/disponen-medidas-adicionales

Resolución de Superintendencia 065-2020/SUNAT. “Amplían medidas para facilitar el cumplimiento de las obligaciones tributarias por declaratoria de emergencia nacional y ampliación del aislamiento social obligatorio", 30 de marzo de 2020. http:// www.sunat.gob.pe/legislacion/superin/2020/065-2020.pdf 
Resolución de Superintendencia 161-2015/SUNAT. “Aprueban nuevo Reglamento de aplazamiento y/o fraccionamiento de la deuda tributaria por tributos internos", 15 de julio de 2015. http://www.sunat.gob.pe/legislacion/superin/2015/161-2015.pdf

Resolución de Superintendencia 190-2015/SUNAT. “Aprueban disposiciones para la aplicación de la excepción que permite a la Sunat otorgar aplazamiento y/o fraccionamiento por el saldo de deuda tributaria de tributos internos anteriormente acogida al artículo $36^{\circ}$ del código tributario”, 17 de julio de 2015. http://www.sunat.gob.pe/ legislacion/superin/2004/indices/indcor.htm

Resolución de Superintendencia 199-2004/SUNAT. “Aprueban el Reglamento de Aplazamiento y/o Fraccionamiento de la Deuda Tributaria”.

SEAP - SUNAT. Sistema Especial de Actualización y Pago de deudas tributarias. www.sunat. gob.pe > legislacion > seap

SUNAT Ley 27681. Ley de reactivacion a traves del sinceramiento de las deudas tributarias (RESIT). www.sunat.gob.pe > legislacion > fraccion > ley27681

Fecha de recepción: 03 de julio de 2020

Fecha de aprobación par 1: 07 de julio de 2020

Fecha de aprobación par 2: 13 de julio de 2020 